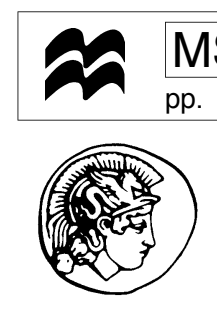

PERGAMON

Lakes, R. S., "Elastic and viscoelastic behaviour of chiral materials", Int. J. of Mechanical Sciences, 43, 1579-1589, June (2001).

International Journal of Mechanical Sciences 00 (2001) 000-000

\title{
Elastic and viscoelastic behavior of chiral materials
}

\author{
Roderic Lakes* \\ Department of Engineering Physics, University of Wisconsin-Madison, 147 Engineering Research Building, \\ 1500 Engineering Drive, Madison, WI 53706-1687, USA
}

Received 13 January 2000; received in revised form 29 August 2000

\begin{abstract}
Chiral materials are not invariant to inversions: there is a distinction between right- and left-handed material. Material properties such as piezoelectricity and pyroelectricity, represented by tensors of odd rank, can only occur in chiral materials. Chiral effects in elasticity cannot be expressed within classical elasticity since the modulus tensor, which is fourth rank, is unchanged under an inversion. We consider effects of chirality in elastic materials described by a generalized continuum representation, specifically Cosserat elasticity. Analysis of several configurations discloses a chiral material to generate reaction moments when compressed as a slab. A chiral plate bent to hyperbolic shape is predicted to exhibit size effects from the Cosserat characteristic length, and a shear force from the chirality. This analysis can be used for the interpretation of experiments on compliant chiral materials, in particular the evaluation of the elastic constants. Viscoelastic chiral solids are examined in the context of the correspondence principle. (C) 2001 Elsevier Science Ltd. All rights reserved.
\end{abstract}

Keywords:

\section{Introduction}

A solid which is not isotropic with respect to inversions is called noncentrosymmetric, acentric, hemitropic, or chiral. Such a solid can be isotropic with respect to direction (coordinate rotations). Chirality is well known in electromagnetics [1]; it gives rise to optical activity in which left or right handed circularly polarized waves propagate at different velocities; applications and recent developments are summarized at the bottom of this section. Chirality, however, has no effect on the classical elastic properties of a material. To demonstrate this, consider the tensorial

\footnotetext{
* Corresponding author. Tel.: + 00-1-608-265-8697; fax: + 00-1-608-263-7451.

E-mail address: lakes@engr.wisc.edu (R.S. Lakes).
} 
Hooke's law

$$
\sigma_{i j}=C_{i j k l} \varepsilon_{k l},
$$

in which $\sigma_{i j}$ is stress, $\varepsilon_{k l}$ is strain and $C_{i j k l}$ is the elastic modulus tensor.

The transformation law for the modulus tensor under coordinate changes is

$$
C_{i j k l}^{\prime}=a_{i m} a_{j n} a_{k o} a_{l n} C_{m n o p}=\frac{\partial x_{m}}{\partial x_{i}} \frac{\partial x_{n}}{\partial x_{j}} \frac{\partial x_{o}}{\partial x_{k}} \frac{\partial x_{p}}{\partial x_{l}} C_{m n o p}
$$

For an inversion, the transformation matrix is just the negative of a Kronecker delta

$$
\begin{aligned}
& a_{i m}=-\delta_{i m}, \\
& C_{i j k l}^{\prime}=(-1) \delta_{i m}(-1) \delta_{j n}(-1) \delta_{o k}(-1) \delta_{p l} C_{\text {mnop }}, \\
& C_{i j k l}^{\prime}=(-1)^{4} C_{i j k l}=C_{i j k l} .
\end{aligned}
$$

So the classical elastic modulus tensor is unchanged by chirality. Similarly, other material properties, such as density and thermal expansion which are describable by tensors of even rank, are unchanged by chirality.

Tensor properties of odd rank are zero if there is inversion symmetry, and can only be nonzero if there is chirality. Examples include piezoelectricity, governed by a third rank tensor, and strain gradient elastic theories which are governed by a fifth rank tensor. Some materials behave as generalized continua which allow more freedom than classical elasticity. For example, Cosserat elasticity [2] also referred to as micropolar elasticity [3] has a characteristic length scale. The non-classical behavior is influenced by chirality [4].

Materials may exhibit chirality on the atomic scale, as in quartz and in biological molecules. Materials may also exhibit chirality on a larger scale, as in composites with helical or screw-shaped inclusions. Such materials can exhibit odd rank tensor properties such as piezoelectric response. They may exhibit torsional deformation when stretched. A material such as aluminum crystallizes in a face-centered cubic lattice which has a center of symmetry, hence no chirality.

Chirality of materials is presently probed by optical methods involving observation of rotation of the plane of polarization of polarized light [5,6]. Recently, a chiral composite was developed, based on a periodic array of conducting nonmagnetic split ring resonators and continuous wires, that exhibits a frequency region in the microwave regime with simultaneously negative values of effective permeability and permittivity [7]. In such a medium, such phenomena as the Doppler effect, Cherenkov radiation, and even Snell's law are inverted. Chiral thin films made of nanometer scale helical columns exhibit controllable optical rotation [8]. Transparent solids, clear single crystals, or solutions may be studied optically, but polycrystalline aggregates, composites, foams, and other opaque materials are not amenable to optical methods. Chiral mechanics, described in this article, may be of use as an alternate probe. Chiral mechanics may also be of use as a guide in the synthesis of high damping viscoelastic composites, as well as novel materials for use in actuators which couple axial and twisting motion. 


\section{Cosserat elasticity}

\subsection{Isotropic solids}

Cosserat solids [2,3] have an extra kinematical degree of freedom: the micro-rotation $\phi$ of points in addition to the translation $u$ considered in classical elasticity. There is a corresponding dynamical degree of freedom, the couple stress $m_{k l}$, or couple per unit area. The constitutive equations for a linear isotropic Cosserat solid are as follows [3]:

$$
\begin{aligned}
& \sigma_{k l}=\lambda \varepsilon_{r r} \delta_{k l}+(2 \mu+\kappa) \varepsilon_{k l}+\kappa \varepsilon_{k l m}\left(r_{m}-\phi_{m}\right), \\
& m_{k l}=\alpha \phi_{r, r} \delta_{k l}+\beta \phi_{k, l}+\gamma \phi_{l, k},
\end{aligned}
$$

where $\sigma_{k l}$ is the force stress, which is a symmetric tensor in classical elasticity but it is asymmetric in Eq. (6). $m_{k l}$ is the couple stress, $\varepsilon_{k l}=\left(u_{k, l}+u_{l, k}\right) / 2$ is the small strain, $u_{k}$ is the displacement, and $e_{k l m}$ is the permutation symbol. The microrotation $\phi_{k}$ in Cosserat elasticity is kinematically distinct from the macrorotation $r_{k}=\left(e_{k l m} u_{m, l}\right) / 2$ obtained from the displacement gradient.

In three dimensions, the isotropic Cosserat elastic solid requires six elastic constants $\lambda, \mu, \alpha, \beta, \gamma$, and $\kappa$ for its description. A comparison of symbols used by various authors was presented by Cowin [9]. The following technical constants derived from the tensorial constants are more beneficial in terms of physical insight. These are $[3,9,10]$

$$
\begin{aligned}
& \text { Young's modulus } E=(2 \mu+\kappa)(3 \lambda+2 \mu+\kappa) /(2 \lambda+2 \mu+\kappa), \\
& \text { shear modulus } G=(2 \mu+\kappa) / 2, \\
& \text { Poisson's ratio } v=\lambda /(2 \lambda+2 \mu+\kappa), \\
& \text { characteristic length for torsion } \ell_{\mathrm{t}}=[(\beta+\gamma) /(2 \mu+\kappa)]^{1 / 2}, \\
& \text { characteristic length for bending } \ell_{\mathrm{b}}=\left[(\gamma / 2(2 \mu+\kappa)]^{1 / 2},\right. \\
& \text { coupling number } N=[\kappa / 2(\mu+\kappa)]^{1 / 2} \text {, and } \\
& \text { polar ratio } \Psi=(\beta+\gamma) /(\alpha+\beta+\gamma) .
\end{aligned}
$$

When $\alpha, \beta, \gamma, \kappa$ vanish, the solid becomes classically elastic. The case $N=1$ (its upper bound) is known as 'couple stress theory' [11].

Isotropic Cosserat elasticity has the following consequences.

(i) A size-effect is predicted in the torsion of circular cylinders of Cosserat elastic materials. Slender cylinders of diameter of the same order of magnitude as $\ell_{\mathrm{t}}$ appear more stiff then expected classically [10]. A similar size effect, governed by $\ell_{\mathrm{b}}$, is also predicted in the bending of plates [10] and of round rods [12]. No size effect is predicted in tension.

(ii) The stress concentration factor for a circular hole, is smaller than the classical value, and small holes exhibit less stress concentration than larger ones [13].

(iii) Dilatational waves propagate non-dispersively, i.e. with velocity independent of frequency, in an unbounded isotropic Cosserat elastic medium as in the classical case. Shear waves propagate dispersively in a Cosserat solid [3]. A new kind of wave associated with the micro-rotation is predicted to occur in Cosserat solids. 
(iv) The mode structure of vibrating Cosserat bodies is modified from that of classical elastic bodies [11].

(v) The range in Poisson's ratio is from -1 to +0.5 , the same as in the classical case [5].

Cosserat-type theories were intensively developed in the 1960s and 1970s. Metals [14] and a particulate composite [10] were experimentally found to behave entirely classically. More recently, such theories have been used in interpreting observations in geomechanics [15] and in biomechanics as discussed in Section 4.

\subsection{Chiral elastic solids}

For a chiral Cosserat elastic solid which is isotropic with respect to direction, the constitutive equations are as follows [4]. Such materials are also called hemitropic [16], noncentrosymmetric or acentric. Chirality has no mechanical effect in classical elasticity.

$$
\begin{aligned}
& \sigma_{k l}=\lambda \varepsilon_{r r} \delta_{k l}+(2 \mu+\kappa) \varepsilon_{k l}+\kappa \varepsilon_{k l m}\left(r_{m}-\phi_{m}\right)+C_{1} \phi_{r, r} \delta_{k l}+C_{2} \phi_{k, l}+C_{3} \phi_{l, k}, \\
& m_{k l}=\alpha \phi_{r, r} \delta_{k l}+\beta \phi_{k, l}+\gamma \phi_{l, k}+C_{1} \varepsilon_{r r} \delta_{k l}+\left(C_{2}+C_{3}\right) \varepsilon_{k l}+\left(C_{3}-C_{2}\right) \varepsilon_{k l m}\left(r_{m}-\phi_{m}\right) .
\end{aligned}
$$

The constants $\lambda$ and $(2 \mu+\kappa)=2 G$ have the same meaning as in classical elasticity; $\alpha, \beta, \gamma$ and $\kappa$ are as in isotropic Cosserat elasticity; and $C_{1}, C_{2}$ and $C_{3}$ represent the effect of chirality. A somewhat different form was developed from the general Mindlin microstructure elasticity [17] by Weitsman [16]. In chiral materials qualitatively new phenomena are predicted. A rod under tensile load deforms in torsion [4]. Wave speed for transverse circularly polarized waves depends on the sense of polarization. This leads to rotation of the principal plane of elliptically polarized transverse waves $[18,19]$. Examples of chiral materials include crystalline materials such as sugar which are chiral on an atomic scale, as well as composites with helical inclusions or spiraling fibers. A schematic micro-element of such a composite is shown in Fig. 1.

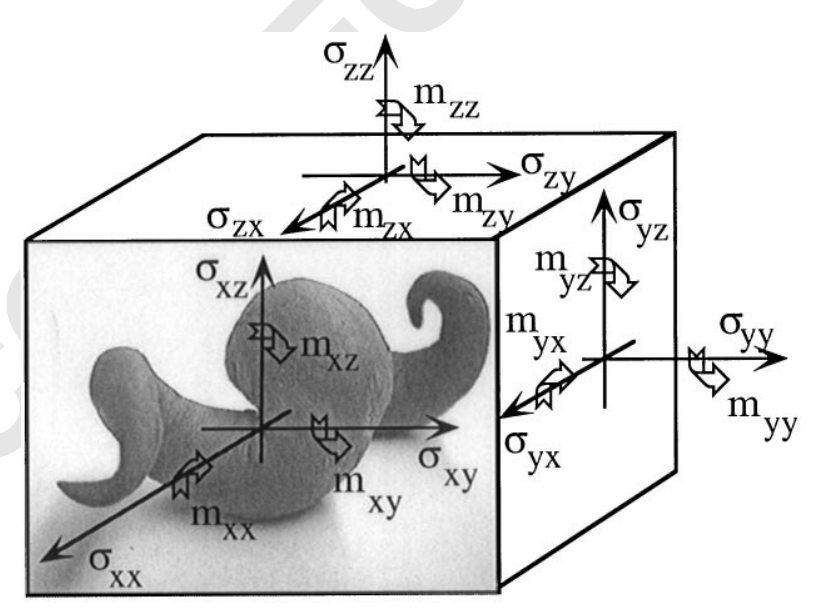

Fig. 1. Differential element of a Cosserat continuum showing elements of stress $\sigma$ (force/area) and couple stress $m$ (moment/area). Also shown is a schematic chiral inclusion in a composite. 


\section{Deformation of chiral solids}

\subsection{Deformation of a chiral slab}

Consider a slab of chiral elastic material compressed in the $z$ direction, and bounded by surfaces $z= \pm H$. Assume one component of displacement $u_{z}(z)$, and one component of microrotation, $\phi_{z}(z)$ to be nonzero. The equilibrium equations [3] for stress $\sigma$,

$$
\sigma_{j k, j}=0
$$

give, for $k=3$

$$
(\lambda+2 \mu+\kappa) \frac{\partial^{2} u_{z}}{\partial z^{2}}+\left(C_{1}+C_{2}+C_{3}\right) \frac{\partial^{2} \phi_{k}}{\partial z^{2}}=0 .
$$

The equilibrium equations for couple stress $m$,

$$
m_{j k, j}+e_{k m n} \sigma_{m n}=0
$$

give, for $k=3$,

$$
\left(1-K_{0}^{2}\right) \frac{\partial^{2} u_{z}}{\partial z^{2}}+\frac{2 \kappa}{\alpha+\beta+\gamma} \phi_{k}=0
$$

with a coupling coefficient defined as

$$
K_{0}^{2}=\frac{\left(C_{1}+C_{2}+C_{3}\right)^{2}}{(\lambda+2 \mu+\kappa)(\alpha+\beta+\gamma)} .
$$

The solution for the microrotation field is

$$
\begin{aligned}
& \phi_{z}(z)=\phi_{0} \sinh p z, \text { with } \\
& p=\sqrt{\frac{2 \kappa}{\alpha+\beta+\gamma} \frac{1}{1-K_{0}^{2}} .}
\end{aligned}
$$

In view of Eq. (18) and the fact that in the absence of chirality we have classical deformation, the displacement field is

$$
u_{z}(z)=e z-\frac{C_{1}+C_{2}+C_{3}}{\lambda+2 \mu+\kappa} \phi_{0} \sinh (p z)
$$

with $\phi_{0}$ to be determined from the boundary conditions.

Several boundary conditions may be considered as follows:

Case 1: $\phi_{z}(z)=0$ at $z= \pm H$ : glued.

This corresponds to a glued or built-in condition at the top and bottom surfaces. Since $\sinh p z \neq 0$ for $z \neq 0, \phi_{z}(z)=0$ everywhere, so $u_{z}(z)=e z$ with $e$ as a strain. The stress is

$$
\sigma_{z z}=(\lambda+2 \mu+\kappa) e .
$$


The axial couple stress is

$$
m_{z z}=\left(C_{1}+C_{2}+C_{3}\right) e .
$$

Therefore a reaction torque is generated via the chiral degrees of freedom in the material. This torque could be readily detected with a tension-torsion test machine.

The stress on the lateral constraint is

$$
\sigma_{x x}=\lambda e .
$$

Moreover, the couple stress on the lateral constraint is

$$
m_{z z}=C_{1} e .
$$

Case 2: $m_{z z}=0$ at $z= \pm H:$ greased.

This corresponds to lubricated or greased compression platens which allow the micro-rotational degrees of freedom to unwind at the surface. There is no reaction torque. Application of the boundary condition gives

$$
\phi_{0}=-e \frac{\left(C_{1}+C_{2}+C_{3}\right) /(\alpha+\beta+\gamma)}{1-K_{0}^{2}}\left\{\frac{1}{p \cosh p H}\right\} .
$$

Substitution of $\phi_{0}$ in Eq. (24) and using Eq. (21) gives the surface displacement

$$
u_{z}(H)=e\left[H+\frac{K_{0}^{2}}{1-K_{0}^{2}} \frac{1}{2} \tanh (p H)\right]
$$

Weitsman [16] obtained a similar form with two chiral elastic constants. The physical interpretation of $p$, which has dimensions of inverse length, is elucidated by expressing it in terms of the characteristic length $\ell_{\mathrm{t}}$ defined in Eq. (11), the coupling number $N$ defined in Eq. (13), the polar ratio $\Psi$ defined in Eq. (14), and the chiral coupling coefficient defined in Eq. (21).

$$
p^{2}=\frac{2}{1-K_{0}^{2}} \Psi \frac{1}{\ell_{\mathrm{t}}^{2}} \frac{N^{2}}{1-N^{2}} .
$$

The compressive stress is as follows. Terms containing $\phi_{z}(z)$ sum to zero.

$$
\sigma_{z z}=(\lambda+2 \mu+\kappa) e .
$$

The chirality does not contribute to the compressional stress. The apparent compressional stiffness is affected by chirality since the axial strain changes. The reduction in stiffness depends on the characteristic length and tends to zero as the slab thickness $2 H$ becomes much larger than the characteristic length.

\subsection{Bending of a chiral plate}

Consider bending of a chiral elastic plate of thickness $h$ and edge length $L$ to a hyperboloid shape. The displacement field is:

$$
u_{x}=-\frac{1}{2 R}\left(z^{2}-y^{2}\right), \quad u_{y}=-\frac{x y}{R}, \quad u_{z}=-\frac{x y}{R} .
$$


The microrotation field is

$$
\phi_{x}=0, \quad \phi_{y}=-\frac{z}{R}, \quad \phi_{z}=-\frac{y}{R}
$$

with $R$ as a radius of curvature and $x$ as the thickness direction of the plate. The equilibrium equations are satisfied by this field. The free surfaces $x=$ constant are free of stress and couple stress as required for an edge-loaded plate. The edge stress $\sigma_{y y}=(2 \mu+\kappa)(x / R)$ gives rise to a bending moment $(2 \mu+\kappa) h^{3} L / 12 R$ as in classical elasticity. The edge couple stress $m_{y z}=-(\beta+\gamma) / R$ gives rise to an additional bending moment $(\beta+\gamma) h L / R$, which is responsible for a size effect in stiffness. Such size effects are typical in Cosserat elasticity. Chirality has no effect upon the stiffness in this example, but a shear stress $\sigma_{y z}=-\left(C_{2}+C_{3}\right) / R$ must be applied at the edges to maintain the hyperboloid shape of the plate. Moreover, self-equilibrated distributions of couple stress $m_{y y}=-\left(C_{2}+C_{3}\right)(x / R)$ and $m_{z z}=\left(C_{2}+C_{3}\right)(x / R)$ appear at the edges.

\subsection{Tension of a chiral rod}

The displacement field of a chiral elastic rod of circular cross section, stretched axially to strain $\varepsilon$ and free to twist [4], in cylindrical coordinates is

$$
u_{z}=\varepsilon z, u_{r}=-\varepsilon\left(v_{0} r+\frac{A_{9}\left(C_{1}+C_{2}+C_{3}\right)}{\varepsilon(\lambda+2 \mu+\kappa)} I_{1}(p r)\right), \quad u_{\theta}=b_{0} r z .
$$

The microrotation field is

$$
\phi_{z}=b_{0} z, \quad \phi_{r}=A_{9} I_{1}(p r)-\frac{1}{2} b_{0} r, \quad \phi_{\theta}=0
$$

with $I_{1}$ as the modified Bessel function of the first kind and $b_{0}, v_{0}$ and $A_{9}$ as constants.

A tensile strain $\varepsilon$ in the $z$ direction gives rise to a torsional deformation with $b_{0}$ as the twist. The Poisson effect is not uniform as shown by the $A_{9}$ term in $u_{r}$. This is an exact solution, but the expression for the twisting response to stretch is a complicated function of all nine elastic constants.

\subsection{Viscoelasticity of chiral materials}

As in the case of classical elasticity, the elastic-viscoelastic correspondence principle may be applied to the constitutive equations and to the elastic solutions derived from them. In the time domain, each elastic constant is replaced by its s-multiplied Laplace transform; the solution for the corresponding viscoelastic problem is obtained by inverse Laplace transformation. In the frequency domain, each elastic constant is replaced by a complex function of frequency. The imaginary part of a modulus term is related to energy dissipation; in passive materials it must be nonnegative. It is simpler to work with the correspondence principle in the frequency domain since the equations are algebraic rather than integral. Cross properties such as Poisson's ratio, piezoelectric coefficients, and the chiral terms $C_{1}$ can be positive or negative in the elastic case. They can have positive or negative imaginary parts in the viscoelastic case. 

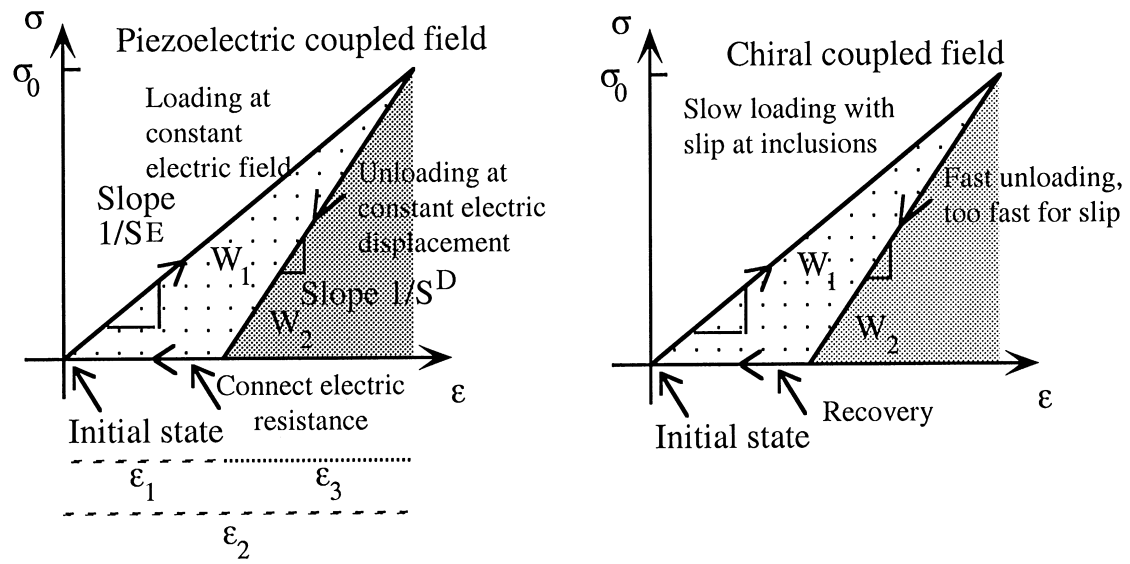

Fig. 2. Dissipation of mechanical energy by coupled field process. Left: conversion of mechanical energy to electrical energy by a piezoelectric material, adapted from Ref. [28]. Energy densities $W$ are shown as shaded areas. $W_{1}$ represents the work per volume of material done by the material on the electrical load, while $W_{1}+W_{2}$ is the maximum energy per unit volume stored in the material at maximum stress. Right: conversion of mechanical energy from axial to rotational form in a chiral elastic solid.

The constitutive equations of chiral materials are of a coupled-field type. If one of the field variables undergoes relaxation, the overall stress-strain relation becomes viscoelastic [20]. Coupled fields can be of use in making new kinds of viscoelastic materials. For example, piezoelectric materials [21] combined with electrical resistance or resistivity have been used where both high stiffness and high mechanical damping are required. The freedom of coupled fields allows conversion of energy as shown in Fig. 2. Chiral composites offer potential as damping materials provided that the chiral degrees of freedom, perhaps embodied as screw or helical inclusions, can relax. The strength of the viscoelastic response due to coupled fields is limited by the dimensionless coupling coefficient with range from zero to one; it describes the degree to which the fields are coupled. In piezoelectric materials the largest coupling coefficient is about 0.7. This is more favorable than the coupling in thermoelastic materials. Some simple centrosymmetric composite microstructures which give rise to high stiffness and high damping have been studied [22]. Referring to Eq. (30), if the coupling coefficient were to approach 1, a large reduction in stiffness could occur with time, corresponding in the frequency domain to a high mechanical damping. However the maximum coupling coefficient for chiral composites is not known, and the potential for viscoelastic composites remains to be explored. Chirality is a subject for future study in this context.

\section{Guidelines for experiments}

Isotropic structured materials have been studied as Cosserat solids, and all six Cosserat elastic constants have been determined for several foam materials $[23,24]$. The moduli $E$ and $G$ were revealed by asymptotic rigidity measurements on large specimens. The characteristic lengths $\ell_{\mathrm{t}}$ and 
$\ell_{\mathrm{b}}$ were determined by measurements of rigidity as a function of diameter for cylindrical specimens in torsion and bending. The dimensionless ratios $N$ and $\Psi$ were inferred from the asymptotic form of the size effects for small specimens in torsion and bending. Similar size effects have been observed in human bone; the effective shear modulus appeared more than four times larger in slender specimens than in thick ones [25]. The predictive power of generalized continuum mechanics is illustrated by the fact that elastic constants derived from size effect measurements allow one to correctly predict strain distributions in bone [26].

As for chiral solids, the slab modality is most appropriate for compliant materials such as foams and rubbery polymers and polymer composites. The reason is that stiffer materials are more difficult to constrain laterally; moreover the structural stiffness of the squat specimen geometry may be excessive in comparison with test machine stiffness. Constrained compression experiments on slabs are more difficult than tension, bending, or torsion tests on slender rods. For slabs one must take care that the assumed boundary conditions correspond in detail to the reality of the experiment since one cannot appeal to Saint-Venant's principle.

The classical elastic constants $E$ and $G$ are obtained via tests on specimens much larger than the characteristic length. They may be obtained directly by conventional tension and torsion tests upon rods or inferred from the Lamé elastic constants extracted from compression tests on slabs.

In the glued slab compression test, measurement of $m_{z z}$ by a torque sensor in the compression platen gives $\left(C_{1}+C_{2}+C_{3}\right)$ via Eq. (26). By measuring the moment due to $m_{x x}$ in the side constraint by a torque sensor, one determines $C_{1}$ via Eq. (28). Since couple stress has dimensions of stress times length, the larger the characteristic length scale associated with the material, the larger the couple stress. Following Eq. (21), the chiral coupling coefficient is also involved in the magnitude of couple stresses arising from chirality. Since it is difficult to measure a small torque in the presence of a large force, materials which are chiral on the atomic or molecular scale are not suitable for characterization by slab compression.

In the lubricated slab compression test, measurement of $\sigma_{x y}$ by a shear force sensor embedded in the side constraint gives $\kappa$ from $\sigma_{x y}=-\kappa \phi_{z}$ via Eq. (15), hence $N$ via Eq. (13). Similarly, Eq. (16) gives $m_{x x}=\alpha \mathrm{d} \phi_{z} / \mathrm{d} z+C_{1} \mathrm{~d} u_{z} / \mathrm{d} z$. Since $C_{1}$ has been determined above, measurement via a torque sensor of the moment due to $m_{x x}$ gives $\alpha$. Measurement of compressional stiffness versus specimen thickness gives $p$ and $K_{0}^{2}$ in Eq. (28); from Eq. (29) and the above constants, $\ell_{\mathrm{t}}$ and $\Psi$ and may be calculated. The remaining characteristic length $\ell_{\mathrm{b}}$ is not revealed by this type of experiment. Therefore the slab compression experiments provide eight of the nine elastic constants of the chiral material.

The plate bend test provides the shear modulus $G=(2 \mu+\kappa) / 2$ and by size effects in the stiffness, the characteristic length $\ell_{\mathrm{t}}=\left[(\beta+\gamma) /(2 \mu+\kappa]^{1 / 2}\right.$. Measurement of the shear force required to maintain the hyperboloid shape gives $C_{2}+C_{3}=-R \sigma_{y z}$.

The rod tension test is simple experimentally, and is suitable for stiff materials as well as compliant ones. Observation of a twisting deformation in response to axial load demonstrates clearly the chirality of a material; some such experiments have been conducted on bone [27] However interpretation in terms of elastic constants is difficult since the twisting response to stretch depends upon all nine elastic constants in a complicated way. Even so, great sensitivity is possible since end rotation can be measured with great precision. As a result, an atomic scale characteristic length would give rise to a measurable twist in a stretched chiral fiber of macroscopic size. 


\section{Conclusions}

Chiral material generate reaction moments when compressed as a slab. Constrained compression experiments can be used to extract eight of the nine elastic constants of a directionally isotropic, chiral solid. Chiral viscoelastic solids may be understood by applying the correspondence principle to the solutions for the corresponding elastic solids.

\section{References}

[1] Post EJ. Formal structure of electromagnetics. North-Holland, Amsterdam, 1962.

[2] Mindlin RD. Stress functions for a Cosserat continuum. International Journal of Solids and Structures 1965;1:265-71.

[3] Eringen AC. Theory of micropolar elasticity. In: Liebowitz H, editor. Fracture vol. 1. NewYork: Academic Press, 1968. p. 621-729.

[4] Lakes RS, Benedict RL. Noncentrosymmetry in micropolar elasticity. International Journal of Engineering Science 1982;29:1161-7.

[5] Lowry TM. Optical rotatory power. London: Longmans and Green, 1935.

[6] Barron LD. Molecular light scattering and optical activity. Cambridge: Cambridge University Press, 1982.

[7] Smith DR, Padilla WJ, Vier DC, Nemat-Nasser SC, Schultz S. Composite medium with simultaneously negative permeability and permittivity. Physical Review Letters 2000;84:4184-7.

[8] Robbie K, Brett MJ, Lakhtakia A. Chiral sculpted thin films. Nature 1996;384:616.

[9] Cowin SC. Stress functions for Cosserat elasticity. International Journal of Solids and Structures 1970;6:389-98.

[10] Gauthier RD, Jahsman WE. A quest for micropolar elastic constants. Journal of Applied Mechanics 1975;42:369-74.

[11] Mindlin RD, Tiersten HF. Effect of couple stresses in linear elasticity. Archive for Rational Mechanics and Analysis 1962;11:415-48.

[12] Krishna Reddy GV, Venkatasubramanian NK. On the flexural rigidity of a micropolar elastic circular cylinder. Journal of Applied Mechanics 1978;45:429-31.

[13] Mindlin RD. Effect of couple stresses on stress concentrations. Experimental Mechanics 1963;3:1-7.

[14] Ellis RW, Smith CW. A thin plate analysis and experimental evaluation of couple stress effects. Experimental Mechanics 1968;7:372-80.

[15] Dai C, Mühlhaus HB, Meek J, Duncan Fama ME. Modelling of blocky rock masses using the Cosserat method. International Journal of Rock Mechanics and Mining Sciences and Geomechanical Abstracts 1996;33:425-32.

[16] Weitsman Y. Initial stresses and skin effects in a hemitropic Cosserat continuum. Journal of Applied Mechanics 1967;34:160-4.

[17] Mindlin RD. Micro-structure in linear elasticity. Archive for Rational Mechanics Analysis 1964;16:51-78.

[18] Lakhtakia A, Varadan VK, Varadan VV. Elastic wave propagation in noncentrosymmetric, isotropic media: dispersion and field equations. Journal of Applied Physics 1988;64:5246.

[19] Lakhtakia A, Varadan VK, Varadan VV. Reflection of elastic plane waves at a planar achiral-chiral interface. Journal of Acoustical Soceity of America 1990;87:2314-8.

[20] Nowick AS, Berry BS. Anelastic relaxation in crystalline solids. New York: Academic Press, 1972.

[21] Forward RL. Electronic damping of vibrations in optical structures. Journal of Applied Optics 1979;18:690-7.

[22] Brodt M, Lakes RS. Composite materials which exhibit high stiffness and high viscoelastic damping. Journal of Composite Materials 1995;29:1823-33.

[23] Lakes RS. Experimental microelasticity of two porous solids. International Journal of Solids and Structures 1986;22:55-63.

[24] Anderson WB, Lakes RS. Size effects due to Cosserat elasticity and surface damage in closed-cell polymethacrylimide foam. Journal of Materials Science 1994;29:6413-9.

[25] Lakes RS. On the torsional properties of single osteons. Journal of Biomechanics 1995;28:1409-10. 


\section{MS 858}

[26] Park HC, Lakes RS. Cosserat micromechanics of human bone: strain redistribution by a hydration-sensitive constituent. Journal of Biomechanics 1986;19:385-97.

[27] Lakes RS. Is bone elastically noncentrosymmetric? Proceedings of the 34th ACEMB, Houston, September 1981. p. 286.

[28] IEEE Standard on Piezoelectricity, IEEE 176-1978; Institute of Electrical, Electronics Engineers, New York, 1978. 